\title{
Benefits of prolonged-release pirfenidone plus standard of care treatment in patients with advanced liver fibrosis: PROMETEO study
}

\author{
Jorge Luis Poo ${ }^{1,2}$ - Aldo Torre ${ }^{1,3}$. Juan Ramón Aguilar-Ramírez ${ }^{1}$ Mauricio Cruz ${ }^{1}$ Luis Mejía-Cuán ${ }^{1}$ - Eira Cerda ${ }^{1}$. \\ Alfredo Velázquez ${ }^{1}$. Angélica Patiño ${ }^{1}$ - Carlos Ramírez-Castillo ${ }^{1}$. Laura Cisneros ${ }^{1,2}$. Francisco Bosques-Padilla ${ }^{1,2}$. \\ Larissa Hernández $^{7} \cdot$ Frida Gasca $^{1} \cdot$ Francisco Flores-Murrieta $^{4} \cdot$ Samuel Treviñoo ${ }^{5}$. Graciela Tapia ${ }^{6}$. \\ Juan Armendariz-Borunda ${ }^{2,7} \cdot$ Linda E. Muñoz-Espinosa ${ }^{1,8}$
}

Received: 10 January 2020 / Accepted: 22 June 2020 / Published online: 19 August 2020

(c) The Author(s) 2020

\begin{abstract}
Background and aims Pirfenidone (PFD), an oral antifibrotic drug, has been authorized by the EMA and FDA for treatment of idiopathic pulmonary fibrosis. Few studies have addressed its use in advanced liver fibrosis (ALF). We evaluated a prolonged-release formulation (PR-PFD) plus standard of care on disease progression in ALF.

Methods 281 ALF patients from 12 centers receiving PR-PFD (600 mg bid) were screened; 122 completed 1 year of treatment. Additionally, 74 patients received only standard of care regimen. Average age was $64 \pm 12$ years, $58 \%$ female. $43.5 \%$ had fatty liver disease (NAFLD), 22.5\% viral hepatitis C (VHC), 17\% autoimmune hepatitis (AIH), and 17\% alcoholic liver disease (ALD). Baseline fibrosis was F4 in $74 \%$ and F3 in 26\%. Antifibrotic effects were assessed by transient elastography $\left(\right.$ Fibroscan $\left.^{\circledR}\right)$ and Fibro Test ${ }^{\circledR}$ (FT); Cytokines and PFD plasma levels were tracked and quality of life evaluated.

Results We found a significant reduction in fibrosis in 35\% of PR-PFD patients and only in $4.1 \%$ in non PR-PFD patients. Child-Pugh score improved in 29.7\%. Biochemical values remained stable; $40.6 \%$ and $43.3 \%$ decreased ALT or AST, respectively. TGF $\beta 1(\mathrm{pg} / \mathrm{mL})$ levels were lower in PFD-treated patients. PFD serum concentration $(\mu \mathrm{g} / \mathrm{mL})$ was higher $(8.2 \pm 1.7)$ in fibrosis regression profile (FRP) patients compared to fibrosis progression profile (FPP) patients $(4.7 \pm 0.3 \mu \mathrm{g} / \mathrm{mL}, p<0.01)$. $12 \%$ reported transient burning or nausea and 7\% photosensitivity. Quality of life (Euro-Qol scale) improved from $62 \pm 5$ to $84 \pm 3(p<0.001)$ and from $32 \pm 3$ to $42 \pm 2(p<0.008)$ (FACIT scale).
\end{abstract}

Conclusions PR-PFD is efficacious and safe in ALF and associated with promising antifibrotic effects.

Trial registration Clinical trial number: NCT04099407.

Keywords Pirfenidone $\cdot$ Prolonged-release pirfenidone $\cdot$ Fibrosis $\cdot$ Fibrosis-regression $\cdot$ Fibrosis-progression $\cdot$ Cirrhosis . Liver $\cdot$ Antifibrotic $\cdot$ Elastography $\cdot$ Fibrotest

Jorge Luis Poo

consultorio.jpoo@gmail.com

1 Grupo Mexicano para el Estudio de las Enfermedades Hepáticas, Periferico Sur 4349, Local 14, Tlalpan, 14210 Mexico City, Mexico

2 Research Unit, Tecnologico de Monterrey, Monterrey, Mexico

3 Department of Gastroenterology, Instituto Nacional de Ciencias Médicas y Nutrición, Salvador Zubirán, Mexico City, Mexico

4 Instituto Politécnico Nacional y Unidad de Investigación en Farmacología del Instituto Nacional de Enfermedades Respiratorias, Mexico City, Mexico
5 Facultad de Ciencias Químicas, Benemérita Universidad Autónoma de Puebla, Mexico City, Mexico

6 Departmento de Genética y Bioestadística, Universidad Nacional Autónoma de México, Mexico City, Mexico

7 Instituto de Biología Molecular en Medicina y Terapia Génica, Universidad de Guadalajara, Guadalajara, Mexico

8 Hepatology Center, Hospital Universitario, Universidad Autónoma de Nuevo León, Monterrey, Mexico 


\section{Introduction}

Established advanced liver fibrosis (ALF) is considered a progressive disease, induced by several etiologies, with no definitive treatment approved by regulatory authorities. It is well established that, unless the injury is blocked or neutralized, most patients suffer complications due to liver failure and increased risk of hepatocellular carcinoma.

Evidence that liver fibrosis regresses to some extent is well-documented, both in experimental models [1,2] and human liver disease $[3,4]$. For fibrosis to regress, the underlying etiology must be treated [5]. Treatment of the underlying disease and concomitant fibrosis regression is associated with improved clinical outcomes. Fibrosis and even cirrhosis regression is characteristic of virtually all forms of liver disease. As yet, however, there is no established treatment that specifically targets liver fibrosis itself [6].

PFD is an antifibrotic drug [7] approved and with granted commercial authorization in Europe, Japan, the USA, Canada, and Mexico for the treatment of idiopathic pulmonary fibrosis (IPF). In Mexico, it has also been granted approval to treat ALF. PFD provides meaningful clinical effects on reductions in the decrease in forced vital capacity (FVC), 6-min walk distance test (6MWT), and mortality, and has improved the progression-free survival of IPF patients with mild-to-moderate disease [8]. PFD (1800-2400 mg daily) taken with food [9], and under adherence to specific access schemes [10] is well tolerated, the most common side effects being gastrointestinal discomfort and photosensitivity. PFD has a favorable benefit-risk profile and represents a suitable treatment option for patients with mild-to-moderate IPF.

This has made it an appealing candidate for other fibrotic diseases [11]. However, concerns about PFD's potential toxicity must be addressed in diseases that have less ominous prognosis/survival rates. This is particularly pertinent for patients with liver disease, since PFD is mostly metabolized in the liver by cytochrome P450. Armendariz-Borunda et al. have shown that patients with mild ALF treated with standard-release PFD showed a reduction in inflammation and fibrosis of $30 \%$ at 12 months [12] and $67 \%$ at 24 months without significant deleterious side effects [13].

Our study rationale was to evaluate a drug with wellknown effects on multiple organs and tissues, and particularly based on the knowledge that PFD inhibits TGF- $\beta 1$ induced over-expression of collagen type I [11], a common pathway involved in the pathogenesis of liver cirrhosis from different etiologies.

Our study aimed to determine whether therapy with a new prolonged-release formulation of PFD (PR-PFD), specifically designed to reduce toxicity and maintain constant serum blood levels for longer duration, in combination with standard of care therapy, would result in reducing liver fibrosis and offer an even more favorable benefit-risk profile in patients with ALF.

\section{Patients and methods}

This was a real-life, multicenter, open-label, proof-of-concept trial to determine the safety and efficacy of 12 months of treatment with PR-PFD in combination with standard of care treatment in adult patients with chronic liver disease whose fibrosis continued to progress despite abstaining from alcohol (ALD), achieving 1 year sustained virologic response (VHC), or otherwise maintaining stable disease (NAFLD, AIH) including healthy life style recommendations. Fibrosis status was determined on the basis of clinical, biochemical, ultrasound and endoscopic findings compatible with chronic damage and at least two other non-invasive methods that confirmed ALF (F3-F4).

\section{Inclusion and exclusion criteria}

Two hundred and eighty-one patients treated with PR-PFD were identified in 12 medical centers. Patients were excluded if they used hepatotoxic drug, decompensation in the previous 6 months, prior history of malignancy, active infectious processes not of a self-limited nature, hemolysis, alpha-fetoprotein $>100 \mathrm{ng} / \mathrm{L}$, pregnancy, and alcohol or intravenous drug abuse within the previous year, were on active treatment with PR-PFD less than 12 months $(n=88)$ or lacked baseline and final fibrosis measurements $(n=71)$ (Fig. 1).

The study group comprised the remaining 122 patients. 71 were female and 51 male. Etiology was ALD in 21 patients (17\%), AIH in $21(17 \%), \mathrm{HCV}$ in 27 patients (22\%), and NAFLD in 53 (43\%). All HCV patients received previous treatment for virologic disease and still had advanced

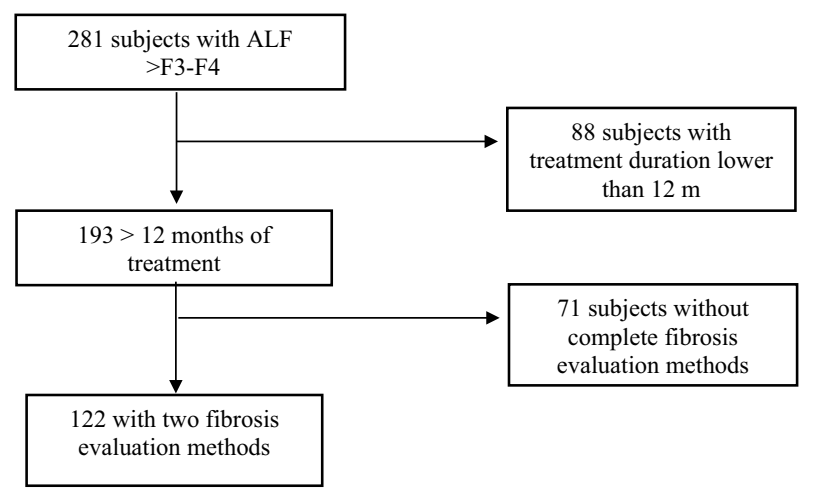

Fig. 1 Inclusion and exclusion criteria 
fibrosis despite sustained viral response (SVR) at 12 months. All patients with $\mathrm{AIH}$ were receiving the lowest possible dose of prednisone and/or azathioprine in order to induce low necroinflammatory activity or biochemical remission. Considering the advance fibrosis stage (F3-F4) of our patient's cohort, none of them presented ALT or AST values higher than twofold ULN.

Patients were 52-76 years of age (mean $64 \pm 12$ years old). All subjects had a complete medical history, lab results, hepatic ultrasound, and upper-gastrointestinal endoscopy at baseline and 12 months (M12) after pharmacological intervention. Patients were negative for hepatocellular carcinoma (HCC) as determined by hepatic ultrasound and had the following lab values: hematocrit $>30 \%$, hemoglobin $>10 \mathrm{~g} / \mathrm{dL}$, platelet count $>30 \times 10^{9} / \mathrm{L}$, white blood cell count $>3 \times 10^{9} / \mathrm{L}$, and serum creatinine level $<1.5 \mathrm{mg} /$ dL. Normal TSH or hypothyroidism under hormone replacement therapy was also required for inclusion. Concomitant disease was detected in 53 patients (43\%) including diabetes mellitus $(n=16)$, systemic hypertension $(n=7)$, dyslipidemia $(n=21)$, osteoarthrosis $(n=11)$, and inflammatory bowel disease $(n=1)$. None of them presented decompensation or required additional treatment during the whole duration of the study.

Ninety were at stage F4 and 32 at F3. Seventy-seven $(85.6 \%)$ of 90 cirrhotic subjects were compensated and 13 decompensated (12 with ascites and 5 with previous esophageal variceal bleeding). All decompensated patients were stable with diuretics. Those with previous variceal hemorrhage received propranolol to achieve $a \geq 20 \%$ reduction in baseline heart rate and completed a variceal band ligation program. Patients with large varices without previous hemorrhage also received betablockers and/or variceal band ligation as primary prophylaxis. None had overt portal systemic encephalopathy. 65 had grade A Child-Pugh scores (72.2\%), 23 had grade B (25.6\%), and 2 had grade C (2.2\%). 27 had a MELD score lower than $9(30 \%)$, and 63 had a MELD of $10-19(70 \%) .43$ patients $(47.7 \%)$ were at stage 1 cirrhosis (without esophageal varices), 30 (33.3\%) at stage 2 (esophageal varices without previous bleeding); 12 (13.3\%) at stage 3 (with clinical- and ultrasound-detected ascites) and $5(5.7 \%)$ stage 4 (previous esophageal variceal bleeding). None suffered gastric variceal bleeding during the study.

For the purpose of evaluation of fibrosis progression evolution, we also included a control group of patients with advanced liver fibrosis $(n=74)$ with standard of care treatment. Baseline demographic and clinical findings compared to patients receiving standard of care plus PF-PFD are depicted in Table 1.

\section{Study design and treatment regimens}

Treatment consisted of $600 \mathrm{mg}$ tablets of PR-PFD (Kitoscell LP $^{\circledR}$ in Mexico and authorized by the local Drugs Agency (COFEPRIS) of the Ministry of Health). Patients were instructed to take medication orally, every $12 \mathrm{~h}$, after breakfast and dinner. All participants were required to adhere to a standard of care that included nutritional support, quarterly medical evaluation to review lab results and adjust medications, bi-annual liver ultrasound, and annual upper-gastrointestinal endoscopy.

\section{Clinical and laboratory evaluation}

Blood counts and liver function tests (bilirubin, albumin, prothrombin time expressed as INR, serum transaminases, glucose, and creatinine) were measured at 12-week intervals. Patient's somatometric measurements (height and body weight), vital signs, and frequency of adverse events (AEs) were recorded. Liver enzymes were scored as stable, improving, or worsening. FT results were analyzed at a central laboratory.

\section{Study end points}

The primary efficacy endpoint was a reduction of fibrosis score by at least $30 \%$ either in FT units or kilo Pascals $(\mathrm{kPa})$ according to hepatic elastography or a reduction of 1 point on the METAVIR scale. Secondary efficacy endpoints included improvement in ALT and/or AST, albumin, serum concentrations of TGFbeta, IL-1 and IL- 6 and endothelin, and Child-Pugh and MELD scores. Worsening MELD was defined as switching from a lower-score to a higher-score and improving as switching from a higher-score to a lowerscore, where Group 1 was MELD $\leq 9$, Group 2 10-19, and Group 3> 20 .

Primary safety endpoints included clinical side effects, blood profile abnormalities, overall survival, and PK findings. Secondary safety endpoints included quality-of-life scores.

\section{Evaluation and classification of fibrosis outcomes}

Fibrosis regression profile (FRP): decreases greater than $30 \%$ in FT score or $30 \%$ in $\mathrm{kPa}$ in liver stiffness measurement (LSM) or decreasing 1 point on the METAVIR score comparing baseline and M12 measurements.

Fibrosis-stabilization profile (FSP): stable FT results or $\mathrm{kPa}$ measurements (variations lower than 30\%) or METAVIR score. 
Table 1 Patient baseline demographic and clinical characteristics

\begin{tabular}{|c|c|c|c|}
\hline Characteristics & Control group $(n=74)$ & $\begin{array}{l}\text { Pirfenidone final group } \\
(n=122)\end{array}$ & $\begin{array}{l}\text { Pirfenidone pre- } \\
\text { selection group } \\
(n=281)\end{array}$ \\
\hline Age (years) & $59 \pm 9$ & $64 \pm 12$ & $62 \pm 12$ \\
\hline Female $[n(\%)]$ & $54(73 \%)$ & $71(58 \%)$ & $138(49 \%)$ \\
\hline \multicolumn{4}{|l|}{ Cirrhosis cause } \\
\hline $\operatorname{HCV}[n(\%)]$ & $30(40.5 \%)$ & $27(22 \%)$ & $71(25.3 \%)$ \\
\hline $\operatorname{HCB}[n(\%)]$ & 0 & 0 & $5(1.8 \%)$ \\
\hline NAFLD $[n(\%)]$ & $27(36.5 \%)$ & $53(43 \%)$ & $113(40.2 \%)$ \\
\hline $\operatorname{ALD}[n(\%)]$ & $8(11 \%)$ & $21(17 \%)$ & $45(16.1 \%)$ \\
\hline Autoimmune $[n(\%)]$ & $9(12 \%)$ & $21(17 \%)$ & $47(16.7 \%)$ \\
\hline \multicolumn{4}{|l|}{ Fibrosis METAVIR score } \\
\hline $\mathrm{F} 3[n(\%)]$ & $3(4 \%)$ & $32(26 \%)$ & $62(22 \%)$ \\
\hline $\mathrm{F} 4[n(\%)]$ & $71(96 \%)$ & $90(74 \%)$ & $219(78 \%)$ \\
\hline \multicolumn{4}{|l|}{ Child-Pugh score } \\
\hline $\mathrm{A}[n(\%)]$ & $68(96 \%)$ & $65(72.2 \%)$ & $208(74 \%)$ \\
\hline $\mathrm{B}[n(\%)]$ & $3(4 \%)$ & $23(25.6 \%)$ & $65(23 \%)$ \\
\hline $\mathrm{C}[n(\%)]$ & 0 & $2(2.2 \%)$ & $8(3 \%)$ \\
\hline \multicolumn{4}{|l|}{ Cirrhosis stage } \\
\hline $1[n(\%)]$ & $39(54.9 \%)$ & $43(47.7 \%)$ & $57(26.4 \%)$ \\
\hline $2[n(\%)]$ & $29(40.8 \%)$ & $30(33.3 \%)$ & $110(50.9 \%)$ \\
\hline $3[n(\%)]$ & $3(4.3 \%)$ & $12(13.3 \%)$ & $35(16.2 \%)$ \\
\hline $4[n(\%)]$ & 0 & $5(5.7 \%)$ & $14(6.5 \%)$ \\
\hline \multicolumn{4}{|l|}{ MELD score } \\
\hline$\leq 9$ & $45(63 \%)$ & $27(30 \%)$ & $130(46 \%)$ \\
\hline $10-19$ & $24(34 \%)$ & $63(70 \%)$ & $143(51 \%)$ \\
\hline$\geq$ a 20 & $2(3 \%)$ & 0 & $8(3 \%)$ \\
\hline
\end{tabular}

Fibrosis progression profile (FPP): increases greater than $30 \%$ in FT score or $\mathrm{kPa}$ or increasing 1 point on METAVIR.

\section{Specific evaluation and classification of biochemical outcomes}

\section{Biochemical markers}

Blood parameters determined after overnight fasting included: albumin, prothrombin time, total bilirubin, ALT, AST, AP, and GGT, measured in fresh serum within $8 \mathrm{~h}$ of collection on an automated biochemistry analyzer (Hitachi 917; Roche Diagnostics). $\alpha 2$ macroglobulin, apolipoprotein-A1, and haptoglobin levels were assayed by nephelometry (Image; Beckman Coulter).

\section{Fibro test $^{\circledR}$}

FT measurements were done on fresh serum, blinded to the clinical data and according to the recommended pre-analytic and analytic methods. The laboratory followed the pre-analytical and analytical recommendations required to obtain the fibrosis marker score FT [14]. FT provides a quantitative estimate of liver fibrosis ranging from 0.00 to 1.00 . The semi-quantitative analysis used predetermined cut-offs equivalent to the standard cut-offs for non-cirrhotic METAVIR stages; for FT: F0 (0-0.28), F1 (>0.28-0.48), F2 (>0.48-0.58) and F3 (>0.58-0.74). Cirrhotic stages were as follows: F4.1 ( $>0.74-0.85)$, F4.2 (>0.85-0.95) and F4.3 (>0.95-1.00). A significant decrease/increase in fibrosis was defined as a decrease/ increase of $30 \%$. This test was not performed on control group patients.

\section{Hepatic elastography}

Transient elastography (TE) was performed according to published recommendations [15] using the Fibro-Scan ${ }^{\circledR}$ $\mathrm{M}$ probe. LSM was expressed in $\mathrm{kPa}$. Only procedures with at least 10 validated measurements, a $>60 \%$ success rate, and an interquartile range $<30 \%$ of the median were considered reliable. The semi-quantitative analysis used predetermined cut-offs equivalent to the standard cut-offs 
for non-cirrhotic METAVIR stages. F0 (0-5 kPa), F1 (> 5-7.1 kPa), F2 (>7.1-9.5 kPa), and F3 (>9.5-12.5 kPa). Cirrhotic stages were as follows: for F4.1 $(>12.5-20 \mathrm{kPa})$, for F4.2 (> 20-50 kPa), and for F4.3 (> 50-75 kPa). A significant decrease/increase in fibrosis was defined as a decrease/increase of $30 \%$ in $\mathrm{kPa}$ units. Patients with ascites were offered real-time shear wave elastography (Aixplorer, Supersonic Imagine), a more reliable evaluation method for these patients [16].

\section{Cytokines and pirfenidone}

Serum concentrations of interleukin 6 (IL-6), transforming growth factor beta (TGF- $\beta 1$ ), endothelin 1 (ET-1), and tumor necrosis factor alpha (TNF- $\alpha$ ) were quantified by ELISA in an automated EIA analyzer Coda Microplate System (Bio-Rad Laboratories, Inc., Hercules, California, USA) [17] and values normalized against serum of healthy volunteers $(N=32)$ with normal liver function and $\mathrm{LSM}<5 \mathrm{kPa}$ (F0) and non-treated cirrhotic patients $(n=31)$ with LSM $>12.5 \mathrm{kPa}$ (F4). A subgroup of 65 PR-PFD treated patients was included for cytokines measurements. PFD plasma levels were measured in fasting conditions and $2 \mathrm{~h}$ after standard breakfast (around $400 \mathrm{kcal}$ ) and medication intake, using an HPLC with UV detection method.

\section{Evaluation of safety profile}

Monitoring for safety and toxicity was performed throughout the study. When necessary, appropriate medical intervention was provided. PR-PFD was suspended in any patient who experienced severe clinical (e.g., photosensitivity) or laboratory toxicity (grade 3, modified Aids Clinical Trials Group graded toxicity scale), until toxicity returned to baseline. PRPFD was permanently discontinued if toxicity persisted or if a patient experienced life-threatening (WHO grade modified ACTG graded toxicity scale) toxicities.

\section{Quality-of-life assessment}

All patients filled out the Euro-Qol Index survey, including the visual analog scale evaluation at baseline and at $12 \mathrm{~m}$. We also incorporated the nonutility-based Short Form-36v2 survey, which provides a detailed profile of health-related quality of life [18].

\section{Statistical data analysis}

Statistical evaluation was performed using IBM SPSS ${ }^{\circledR}$ version 22. Differences in laboratory parameters such as ALT, AST or cytokines were compared between groups using Student's $t$ test or Mann-Whitney $U$ test or oneway ANOVA test when applicable. Given that the data of cytokines and PFD showed a non-normal distribution, they were analyzed by a generalized linear model (GzLM) with maximum likelihood as method of estimation. The marginal means were estimated for each treatment and compared by pairs of groups by Bonferroni test. Significant statistical differences were considered at $p<0.05$. Sample size calculation was based on previous data from our local experience and the following assumptions: (a) mean expected baseline elastography score of $27.4 \mathrm{kPa}$ (b) standard deviation of 15.7; (c) expected estimated-fibrosis reduction rate higher than $30 \%$ (delta); (d) alpha error of 1\%; (e) an accepted beta error of $10 \%$ (power $=90 \%$ ); (f) two tails. The final number of patients required to find a significant difference using the $G$ power statistical program [19] was 61 patients.

\section{Results}

A flowchart of the total study population is shown in Fig. 1. Only 122 participants with ALF (F3-F4) completed studymedication for at least 12 months. As expected in an open, real-life trial, some patients $(n=71)$ did not provide the fibrosis evaluation methods. However, an intention-to-treat (ITT) analysis showed no statistically significant differences between patients included and those who were not included (baseline data in Table 1).

\section{Fibrosis regression}

A significant reduction in LSM, 26.5 $\pm 2.0 \mathrm{kPa}$ versus $21.5 \pm 2.0, p<0.05$ and FT score, $0.79 \pm 0.01$ versus $0.61 \pm 0.02, p<0.0001$ was observed in the total population (Fig. 2). In the PR-PFD group, 35.2\% $(n=43)$ presented a FRP; $46.7 \%$ (57 patients) a FSP; and $18.0 \%$ (22 patients) a FPP. In the control group, only $4.1 \%(n=3)$ presented a FRP; $78.3 \%$ (58 patients) a FSP; and $17.6 .0 \%$ (13 patients) a FPP (Table 2). LSM score increased from $21.6 \pm 0.0 .98 \mathrm{kPa}$ to $24.9 \pm 1.07$.

\section{Clinical outcomes}

One hundred and seven patients finished at Grade A, 15 at Grade B, and 0 at Grade $C ; 8$ improved from Grade B to Grade A and 2 from Grade C to Grade B/Grade A (Table 2). 36 (29.5\%) decreased or improved $(p<0.001)$ and $13(10.7 \%)$ increased or worsened by 1 point, while $73(59.8 \%)$ remained stable.

A higher number ended in MELD score group $1(\leq 9)$, and only $2(1.6 \%)$ progressed to $>20$ (Tables 3 and 4 ); 88 $(72.1 \%)$ remained stable, $16(13.1 \%)$ worsened, and 18 (14.8\%) improved MELD score. 
Liver stifness (kPascals)

a
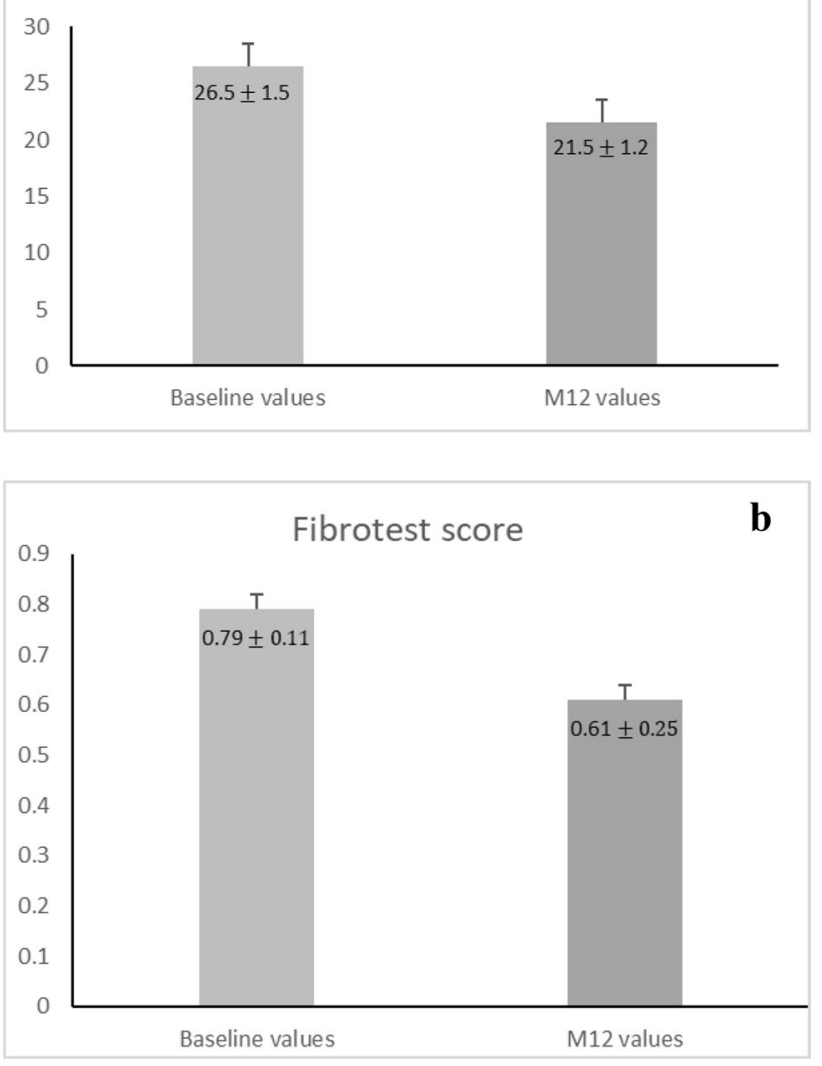

Fig. 2 a Statistical significance was analyzed by Student's $t$ test, $p=0.023$, compared to baseline values. b Statistical significance was analyzed by Student's $t$ test, $p=0.0001$, compared to baseline values

Blood tests evolution remained stable in 75 patients (61.5\%). Only nine patients changed Cirrhosis stages, 7 from Stage 3 to Stage $2(p=0.03)$ and 2 from Stage 3 to Stage 4. (One presented active hematemesis; one had melena with active variceal hemorrhage confirmed by endoscopy.) None had multiple liver-related AE and/or other organ involvement. None suffered gastric variceal bleeding. None presented uncontrolled encephalopathy or uncontrolled ascites. Noteworthy, ALT and AST serum levels remained stable in $53(43.4 \%)$ and $56(45.9 \%)$, respectively; $49(40.2 \%)$ and $53(43.4 \%)$ decreased transaminase serum levels $(p<0.00005)$ and only 20 $(16.4 \%)$ and $13(10.7 \%)$ increased.

Body weight and BMI values decreased at month 12, whereas global biochemical data remained stable (Table 5).

\section{Endoscopic findings}

Absence of esophageal varices improved from 26 patients (21.3\%) to $38(34.9 \%)$ at M12. That is, esophageal varices disappeared in 12 patients. At baseline, $62(50.8 \%)$ had small and 34 (27.9\%) had large varices. In the 109 who accepted endoscopy at M12, 25\% had small varices and $40 \%$ had large varices. Although varices increased in size in 10 , only 2 had variceal hemorrhage.

\section{Liver ultrasound major findings}

Chronic liver disease was confirmed at baseline in 122 patients, 53 patients had splenomegaly and 12 patients had mild ascites. At M12, 68 had splenomegaly and 6 had ascites.

\section{Cytokines serum concentration}

Cytokines levels in the serum of healthy subjects were significantly lower compared to those in PR-PFD-nontreated cirrhotics and our study group (Table 6). TGF$\beta 1$ concentrations were significantly higher in cirrhotic patients without treatment $(99.6 \pm 7.5 \mathrm{pg} / \mathrm{mL})$ compared to control subjects $(46.2 \pm 2.5 \mathrm{pg} / \mathrm{mL}, p<0.01)$ and patients under active treatment with PR-PFD $(61.8 \pm 2.3 \mathrm{pg} / \mathrm{mL}$, $p<0.05)$. Interleukin-6 $(10.5 \pm 1.04$ versus $13.9 \pm 1.7$, $\mathrm{pg} / \mathrm{mL}, p<0.05)$ and endothelin-1 $(17.8 \pm 1.6$ versus $22.1 \pm 3.41, \mathrm{pg} / \mathrm{mL}, p<0.05)$ were also significantly decreased in PR-PFD-treated patients compared to nontreated cirrhotics (Table 6).
Table 2 Liver fibrosis evolution according to elastography

\begin{tabular}{lcl}
\hline & Control group $(n=74)$ & $\begin{array}{l}\text { Pirfeni- } \\
\text { done group } \\
(n=122)\end{array}$ \\
\hline According to liver stiffness by hepatic elastography measurement & \\
Fibrosis progression profile (FPP) $[n(\%)]$ & $13(17.6 \%)$ & $22(18.3 \%)$ \\
Fibrosis-stable profile (FSP) $[n(\%)]$ & $58(78.3 \%)$ & $57(46.7 \%)$ \\
Fibrosis regression profile (FRP) $[n(\%)]$ & $3(4.1 \%)$ & $43(35.2 \%)$ \\
\hline
\end{tabular}

A significant decrease/increase in fibrosis was defined as $30 \%$ of change, compared to baseline values 
Table 3 Child-Pugh class and MELD evolution in F4 patients

\begin{tabular}{llllll}
\hline & \multicolumn{2}{l}{$\begin{array}{l}\text { Control group } \\
(n=71)\end{array}$} & & \multicolumn{2}{l}{$\begin{array}{l}\text { Pirfenidone group } \\
(n=90)\end{array}$} \\
\cline { 2 - 3 } \cline { 6 - 6 } \cline { 5 - 6 } & Baseline & Final & & Baseline & Final \\
\hline Child-Pugh A & $68(97 \%)$ & $69(97 \%)$ & & $65(72.2 \%)$ & $75(83.3 \%)$ \\
Child-Pugh B & $3(4)$ & $2(3 \%)$ & & $23(25.5 \%)$ & $15(16.7 \%)$ \\
Child-Pugh C & $0(0 \%)$ & $0(0 \%)$ & & $2(2.2 \%)$ & $0(0.0 \%)$ \\
Child-Pugh Score & $5.3 \pm 0.6$ & $5.2 \pm 0.5$ & $5.8 \pm 1.2$ & $5.7 \pm 0.9$ \\
MELD risk categories & & & & \\
Score $\leq 9$ & $45(63 \%)$ & $38(54 \%)$ & & $27(30 \%)$ & $33(36.7 \%)$ \\
Score 10-19 & $24(34 \%)$ & $32(45 \%)$ & & $63(70 \%)$ & $55(61.1 \%)$ \\
Score $>20$ & $2(3 \%)$ & $1(1 \%)$ & & $0(0.0 \%)$ & $2(2.2 \%)$ \\
Average & $8.8 \pm 3.0$ & $9.3 \pm 2.6$ & & $10.2 \pm 3.1$ & $10.0 \pm 3.1$ \\
\hline
\end{tabular}

\section{Pharmacokinetic data}

PFD plasma concentrations measured at 12 months and under continuous treatment were $6.7 \pm 0.78 \mu \mathrm{g} / \mathrm{mL}$ preprandial and $8.9 \pm 0.74 \mu \mathrm{g} / \mathrm{mL}$ post-prandial indicating stable medication levels. Notably, FRP patients had higher fasting PDF plasma levels $(8.2 \pm 1.7 \mu \mathrm{g} / \mathrm{L})$ than FSP $(6.2 \pm 1.4 \mu \mathrm{g} / \mathrm{L}, p<0.05)$ or FPP patients $(4.7 \pm 0.3 \mu \mathrm{g} / \mathrm{L}$, $p<0.001)$. Delta values between fasting and 2-h postprandial were lower in FRP patients (Table 7).

\section{Safety profile}

The side effects seen in our study cohort are described in Table 8.

\section{Survival curve}

An intention-to-treat analysis of the total population taking PF-PFD was carried out even if they did not complete the 12-month treatment period or the whole evaluation (281 patients). There were 8 deaths: 4 were not liver related; 1 followed massive esophageal hemorrhage; 3 were due to progressive liver failure. There were 0 deaths in the study group.

\section{Quality of life}

According to 75 self-reported questionnaires, quality-of-life scale improved from $62 \pm 5$ to $84 \pm 3(p<0.001)$ and FACIT scale from $32 \pm 3$ to $42 \pm 2(p<0.008)$.
Table 4 Child-Pugh score and MELD sub-analysis in PR-PFD group

Table 5 Somatometric and biochemical evolution

\begin{tabular}{llllll}
\hline Child-Pugh & Population & Percentage (\%) & MELD & Population & Percentage (\%) \\
\hline Stable score & 73 & 59.8 & Stable & 88 & 72.1 \\
Increased score & 13 & 10.7 & Worsening & 16 & 13.1 \\
Decreased score & 36 & 29.5 & Improving & 18 & 14.8 \\
\hline
\end{tabular}

Significant changes were defined as 1-point increase or 1-point reduction in Child-Pugh score

Worsening was defined as switching from group 1 (score of 6-9) to group 2 (score 10-19) or group 3 (score $>20$ ) and improving as reducing allocation to a lower-score group

\begin{tabular}{|c|c|c|c|c|}
\hline \multirow[t]{2}{*}{ Characteristics } & \multicolumn{2}{|c|}{ Control group $(n=74)$} & \multicolumn{2}{|c|}{ Pirfenidone group $(n=122)$} \\
\hline & Baseline & M-12 & Baseline & M12 \\
\hline Body weight (kg) & $70.1 \pm 7.3$ & $71.2 \pm 6.96$ & $78.7 \pm 17.9$ & $71.5 \pm 11.3$ \\
\hline $\operatorname{BMI}\left(\mathrm{kg} / \mathrm{m}^{2}\right)$ & $27.98 \pm 7,19$ & $28.41 \pm 6.96$ & $31.2 \pm 7.6$ & $28.9 \pm 3.6$ \\
\hline Hemoglobin (g/dL) & $13.6 \pm 1.4$ & $13.7 \pm 1.2$ & $13.9 \pm 2.2$ & $13.6 \pm 1.6$ \\
\hline Leukocytes $\left(\times 10^{3}\right)$ & $5.2 \pm 1.5$ & $4.8 \pm 1.1$ & $4.5 \pm 1.9$ & $4.5 \pm 1.8$ \\
\hline Platelets $\left(\times 10^{3}\right)$ & $128 \pm 5$ & $131 \pm 6$ & $103 \pm 8$ & $101 \pm 8$ \\
\hline Bilirubin (mg/dL) & $1.1 \pm 0.5$ & $1.1 \pm 0.5$ & $1.6 \pm 1.0$ & $1.3 \pm 0.5$ \\
\hline Albumin (g/dL) & $3.8 \pm 0.4$ & $3.9 \pm 0.4$ & $3.6 \pm 0.6$ & $3.5 \pm 0.5$ \\
\hline P.T. (INR) & $1.2 \pm 0.2$ & $1.2 \pm 0.2$ & $1.3 \pm 0.3$ & $1.3 \pm 0.2$ \\
\hline $\operatorname{ALT}(\mathrm{mg} / \mathrm{dL})$ & $63 \pm 4$ & $66 \pm 2$ & $47 \pm 5.0$ & $45 \pm 4.2$ \\
\hline $\operatorname{AST}(\mathrm{mg} / \mathrm{dL})$ & $73 \pm 4$ & $78 \pm 2$ & $58 \pm 5.9$ & $54 \pm 4.0$ \\
\hline Alkaline phosphatase (mg/dL) & $96 \pm 4$ & $95 \pm 4$ & $194 \pm 17.9$ & $187 \pm 19.3$ \\
\hline GGT (mg/dL) & $69 \pm 8$ & $66 \pm 5$ & $124 \pm 15.7$ & $148 \pm 16.9$ \\
\hline
\end{tabular}

Values are mean $\pm \mathrm{SD}$

$N A$ not available data 
Table 6 TGF- $\beta 1$ serum concentrations

\begin{tabular}{lrcl}
\hline Parameters & Healthy subjects $(n=32)$ & $\begin{array}{l}\text { Subjects with cirrhosis without } \\
\text { treatment }(n=31)\end{array}$ & Subjects with fibrosis and treatment $(n=65)$ \\
\hline TGF- $\beta 1(30-60 \mathrm{pg} / \mathrm{mL})$ & $46.2 \pm 2.5$ & $99.6 \pm 7.5^{\mathrm{a}}$ & $61.8 \pm 2.3^{\mathrm{a}, \mathrm{b}}$ \\
IL-6 $(30-60 \mathrm{pg} / \mathrm{mL})^{*}$ & $3.9 \pm 0.7$ & $13.9 \pm 1.7^{\mathrm{a}}$ & $10.5 \pm 1.04^{\mathrm{a}, \mathrm{b}}$ \\
TNF- $\alpha(1.2-15.3 \mathrm{pg} / \mathrm{mL})$ & $8.7 \pm 1.5$ & $25.97 \pm 2.3^{\mathrm{a}}$ & $23.0 \pm 1.06^{\mathrm{a}}$ \\
Endothelin-1 $(1.2-15.3 \mathrm{pg} / \mathrm{mL})$ & $13.8 \pm 2.2$ & $22.1 \pm 3.4^{\mathrm{a}}$ & $17.8 \pm 1.6^{\mathrm{a}, \mathrm{b}}$ \\
\hline
\end{tabular}

Statistical significance was analyzed by a generalized linear model with maximum likelihood as method of estimation and Bonferroni test ${ }^{\text {a }} p<0.01$ compared to healthy subjects

${ }^{\mathrm{b}} p<0.05$ compared to cirrhotic patients, non-treated with pirfenidone

Table 7 Pirfenidone serum concentrations

\begin{tabular}{llll}
\hline Pirfenidone serum levels & $\begin{array}{l}\text { Fibrosis pro- } \\
\text { gression profile } \\
\text { (FPP) }\end{array}$ & $\begin{array}{l}\text { Fibrosis-stable profile } \\
(\text { FSP })\end{array}$ & $\begin{array}{l}\text { Fibrosis regression } \\
\text { profile (FRP) }\end{array}$ \\
\hline Fasting sample $(\mu \mathrm{g} / \mathrm{mL})$ & $4.7 \pm 0.3$ & $6.2 \pm 1.4$ & $8.2 \pm 1.7^{\mathrm{a}, \mathrm{b}}$ \\
2-h post-prandial sample $(\mu \mathrm{g} / \mathrm{mL})$ & $8.2 \pm 0.1$ & $9.0 \pm 1.3$ & $9.3 \pm 1.4$ \\
\hline
\end{tabular}

Statistical significance was analyzed by a generalized linear model with maximum likelihood as method of estimation and Bonferroni test

${ }^{\mathrm{a}} p<0.01$ compared to fibrosis progression profile subjects

${ }^{\mathrm{b}} p<0.05$ compared to fibrosis-stable profile subjects
Table 8 Side effects list

\begin{tabular}{lll}
\hline Characteristics & Pirfenidone final group $(n=122)$ & Pirfenidone pre-selection group $(n=281)$ \\
\hline Nausea & $12(9.8 \%)$ & $37(13.2 \%)$ \\
Dyspepsia & $10(8.2 \%)$ & $30(10.7 \%)$ \\
Diarrhea & $4(3.3 \%)$ & $12(4.2 \%)$ \\
Rash & $9(7.4 \%)$ & $17(6.0 \%)$ \\
Death & $0(0 \%)$ & $8(2.8 \%)$ \\
\hline
\end{tabular}

$N A$ not available data

\section{Discussion}

This first-ever, real-life, multicenter, open-label study shows a significant effect of PR-PFD in reducing ALF. Significant beneficial effects were also noted in several clinical, biochemical, and molecular parameters.

Treatment with a prolonged-release formulation of PR-PFD, in combination with a standard of care regimen, resulted in either a FRP or a FSP in the vast majority $(35 \%$ and $46.7 \%$, respectively), confirming PR-PFD's significant antifibrotic effects in ALF and extending previous findings $[24,25]$. A remarkable difference was observed when compared to only $4.1 \%$ FRP seen in the control group, receiving the standard of care regimen.

While some clinicians used to consider cirrhosis irreversible, there are well-documented cases of reversibility of ALF [3], largely correlated with effective treatment of the particular etiology $[4,5]$. However, in some patients, fibrosis progresses despite effective treatment. Accordingly, there is a need for ongoing research for treatments directed at fibrosis itself.

This has stimulated evaluation of new antifibrotic drugs [6] in hepatic disorders. Promising studies, like those of Armendariz-Borunda et al. [12,13] show reduction in both inflammation and fibrosis with negligible side effects. Nonetheless, more rigorous studies concerning PFD's safety are mandatory, particularly in ALF and given that drug toxicity could increase due to liver impairment.

In our study, PR-PFD was safe and generally well tolerated. Treatment adherence was high, partially explained by patient motivation and a friendly dosing schedule. The type and frequency of $\mathrm{AE}$ were consistent with the known safety profile of PFD $[8,10,11]$, AEs were typically mild or moderate, and none led to permanent treatment discontinuation. No deaths were detected in the 12-month treatment 
population and few overall deaths in the whole cohort of eligible population, only half of those liver related.

Our data on blood plasma levels allowed us to evaluate adherence to treatment and better understand the relationship between plasma levels and fibrosis response. In addition to confirming detectable plasma levels in all participants, we identified higher and significant PFD-plasma levels in FRP patients than in FPP patients, suggesting better drug impregnation in this subgroup of responders. Interestingly, delta values between fasting and 2-h post-prandial levels were similar in FRP patients but different in FPP patients, indicating a possible metabolic effect at the hepatic cytochrome level. In addition, this is the first study that provides information of medication levels in a cirrhotic population.

Because the presence of food significantly reduces the extent of absorption of PFD [20], all participants were instructed to take medication after food to reduce known side effects. The plasma levels we found do not point to an inhibitory effect of absorption in FFP patients, but rather a different hepatic metabolism. Around 60\% of PFD is bound to plasma proteins, especially to albumin [11], which was found in similar levels in FPP and FRP patients. Since up to $50 \%$ of the drug is metabolized by the hepatic CYP1A2 enzyme system to yield 5-carboxy pirfenidone, the inactive metabolite, it is possible that FRP patients have a different enzymatic activity. It is important to keep in mind that our patients were not using fluvoxamine, amiodarone, or propafenone, considered inhibitors of the enzymatic activity of CYP1A2. Similarly, our cohort was not using ciprofloxacin, another CYP1A2 inhibitor. Moderate inducers of CYP1A2, such as tobacco smoking or omeprazole, can reduce the circulating plasma levels of the drug [21]. While none of our patients smoked tobacco, some were taking PPIs. Nevertheless, there was no difference between the FPP and FRP subgroups.

Armendariz-Borunda et al. [22], have suggested that the effectiveness of PFD could be influenced by inherited genetic polymorphisms that increase the risk of developing ALF in some patients. The different PK plasma levels in our study confirm that possibility. Similar clinical trials must consider PFD plasma levels to improve our understanding.

It is assumed that cytokines play central roles in the progression from chronic liver injury to fibrosis/cirrhosis and several pro-inflammatory cytokines (such as IL6, and TNF $\alpha$ ) correlate with disease severity [23]. In addition, evidence suggests that hepatic stellate cells express the highest levels of endothelin receptors that may contribute to hepatic sinusoidal tone and therefore portal hypertension [24]. In our study, TGF $\beta 1$ serum levels were significantly decreased in PR-PFD-treated patients compared to untreated patients. We also found significant reductions in IL6 and endothelin levels. This suggests that cytokine changes may help predict liver fibrosis evolution.
One of the remarkable facts of our study was the inclusion of patients with different ALF etiologies, as it happens in real life. Our population was recruited from both academic hospitals and ambulatory care units in order to mimic the general population profile, which could increase the external validity of our findings. We understand that fibrosis progression may behave differently in relation to etiology of the liver disease, particularly in patients with AIH. However, according to Hartl et al. [25], transient elastography has been shown to be a reliable tool to monitor disease course in AHI.

In relation to higher relative frequency of $\mathrm{HCV}$ among controls (40.5\%) compared to PFD-treated population (22\%), described in Table 1, we consider this finding is explained because our selection criteria required patients with HCVtreatment and at least 1 year of sustained viral response.

A potential limitation is the absence of liver biopsy, a method with many drawbacks, such as sampling error, cost, and risk of complications [26]. This is mitigated, first, because that, too, mimics the population typical of ambulatory care where non-invasive methods of estimating liver fibrosis are frequently used [27]. Second, non-invasive assessments have been developed and adopted in international management guidelines [15]. LSM by different elastographic methods or magnetic resonance has been found to have an excellent correlation with biopsy findings and is increasingly used in clinical trials. Similarly, FT has been extensively evaluated in patients with chronic liver disease due to diverse etiologies and also has a high correlation biopsy findings, particularly in patients with ALF. According to Lau-Corona et al. [28], FT could enhance our ability to assess differences in fibrosis scores in clinical studies and improve our understanding of fibrosis progression. Recently, Mauro et al. [29], evaluated the value of portal pressure, liver stiffness, and enhanced liver fibrosis score measurements to predict fibrosis regression according to paired liver biopsies before and after sustained viral response (SVR) in recurrent $\mathrm{HCV}$ patients. They concluded that the dynamic changes in LSM accurately predict the presence of ALF and clinically significant portal pressure 1 year after SVR and thus can be used as reliable monitoring strategies. Chalasani et al. [30], confirm the utility of non-invasive markers in ALF patients by showing that longitudinal changes in noninvasive measures of fibrosis correlate with improvements in histologic fibrosis and therefore can serve as surrogate end points in clinical trials. Third, we used two validated, non-invasive methods.

It is worth mentioning that we observed an $11 \%$ reduction in body weight throughout the duration of the study in the pirfenidone-treated group. Since diet and exercise are known factors to influence disease progression/regression, and even portal pressure reduction, we consider that controlling these factors should be part of the commitments of the standard of care. 
Another important finding is significant improvements in liver function and liver enzymes. Child-Pugh is considered a strong bedside prognostic indicator in advanced liver disease, often used with MELD to determine need and priority for transplant [18]. Here, the vast majority improved both scores or remained steady, which correlates with an increase in serum albumin, another clinical prognostic indicator.

Contrary to what might be expected regarding possible liver toxicity, no patients' AST/ALT levels increased X5 upper limit of normal (ULN). Rather, we identified a significant decrease in $40.6 \%$ and $43.3 \%$ in ALT and AST serum levels, demonstrating a favorable safety profile. In Angulo et al. study [31], which reports a high proportion of AE in 24 primary sclerosing cholangitis patients, treatment was with standard-release PFD and at a much higher daily dose (2400-3600 mg). Key strategies to prevent and manage common PFD AEs have been described to maximize adherence and prevent AEs [10].

Regarding the suitability of the PFD formulation that we used in our study, PR-PFD offers a lower $C_{\max }$ and longer $T_{\max }$ and half-life pharmacokinetic profile compared to standard-release PFD [20].

PR-PFD association with lower PFD $C_{\max }$ values (Poo $\mathrm{JL}$, et al. Pharmacokinetics of the antifibrotic drug pirfenidone in Child-Pugh A and B cirrhotic patients compared to healthy age-matched controls. Journal of Hepatology 2016;64:S213-S424) may explain the excellent tolerability in our study. Also promising is that the self-assessment questionnaires indicated significant QOL effects, mainly described as improvement in energy and reduction in fatigue.

In conclusion, prolonged-release PFD administration in conjunction with standard of care treatment in patients with advanced liver fibrosis provides beneficial effects that warrant additional studies. Our data suggest that inflammation and liver stiffness could be ameliorated by PFD-treatment, a finding needing confirmation in a placebo-controlled clinical trial.

Acknowledgements We are grateful to Dr. David Kershenobich who thoughtfully and carefully reviewed the manuscript. Additionally, we also acknowledge Erin Ferris and Marc Sander for improving the English language. This study was presented as poster number \#1986, at the Liver Meeting, AASLD, held in San Francisco, California, last Nov-2018.

Author contributions JLP, LEM, AT, JRA, MC, LAMC, EC, AV, AP, $\mathrm{LC}$, and $\mathrm{FB}$ were involved in population recruitment, ambulatory care of the patients, and contributed to the manuscript writing. ST carried out the cytokines measurements and wrote the specific methodology section. FFM carried out the PFD pharmacokinetic measurements and wrote the specific methodoly section. $\mathrm{LH}$ and FG were involved in ambulatory nutritional care and carried out the capturing and integration of a data base with all demographic, clinical and biochemical data; GT participated in protocol planning, sample size calculation, and carried out the biostatistical analysis. JAB reviewed the protocol and implemented recommendations on methodological issues, and made style and syntax changes to the manuscript. JLP led the multicenter group working with PFD, was involved in recruitment and ambulatory care and oversaw the manuscript writing and style. All authors read and approved the final manuscript.

Funding Authors did not receive finalcial support for design, conduction or publication decision of this study. Medifarma provided funds for citykines and pirfenidone serum levels analisis.

\section{Compliance with ethical standards}

Conflict of interest The study was conducted in compliance with the International Standard GCP procedures and the principles of the Declaration of Helsinki. The protocol and informed consent form were approved by a local IRBs and registered in clinical trials.gov (NCT04099407).

Ethical approval All procedures were in accordance with the ethical standards of the responsible committee on human experimentation (institutional and national) and with the Helsinki Declaration of 1975, as revised in 2008 .

Informed consent Informed consent was obtained from all patients for being included in the study.

Open Access This article is licensed under a Creative Commons Attribution 4.0 International License, which permits use, sharing, adaptation, distribution and reproduction in any medium or format, as long as you give appropriate credit to the original author(s) and the source, provide a link to the Creative Commons licence, and indicate if changes were made. The images or other third party material in this article are included in the article's Creative Commons licence, unless indicated otherwise in a credit line to the material. If material is not included in the article's Creative Commons licence and your intended use is not permitted by statutory regulation or exceeds the permitted use, you will need to obtain permission directly from the copyright holder. To view a copy of this licence, visit http://creativecommons.org/licenses/by/4.0/.

\section{References}

1. Perez-Tamayo R. Cirrhosis of the liver: a reversible disease? Pathol Annu 1979;14:183-213

2. Poo JL, Feldmann G, Erlinger S, Braillon A, Gaudin C, Dumont M, et al. Ursodeoxycholic acid limits liver histologic alterations and portal hypertension induced by bile duct ligation in the rat. Gastroenterology 1992;102(5):1752-1759

3. Ellis EL, Mann DA. Clinical evidence for the regression of liver fibrosis. J Hepatol 2012;56:1171-1180

4. Marcellin P, Gane E, Buti M, Afdhal N, Sievert W, Jacobson IM, et al. Regression of cirrhosis during treatment with tenofovir disoproxil fumarate for chronic hepatitis B: a 5-year open-label follow-up study. Lancet 2013;381:468-475

5. Verrill C, Markham H, Templeton A, Carr NJ, Sheron N. Alcoholrelated cirrhosis: early abstinence is a key factor in prognosis, even in the most severe cases. Addiction 2009;104:768-774

6. Bansal MB, Chamroonkul N. Antifibrotics in liver disease: are we getting closer to clinical use? Hepatol Int 2019;13(1):25-39 
7. Macias-Barragan J, Sandoval-Rodríguez A, Navarro-Partida J, Armendáriz-Borunda J. The multifaceted role of pirfenidone and its novel target. Fibrogenesis Tissue Repair 2010;3:16

8. Xaubet A, Serrano-Mollar A, Ancochea J. Pirfenidone for the treatment of idiopathic pulmonary fibrosis. Expert Opin Pharmacother 2014;15(2):275-281

9. Rubino CM, Bhavnani SM, Ambrose PG, Forrest A, Loutit JS. Effect of food and antacids on the pharmacokinetics of pirfenidone in older healthy adults. Pulm Pharmacol Ther 2013;22:279-285

10. Landells LJ, Naidoo B, Robertson J, Clark P. NICE guidance on pirfenidone for treating idiopathic pulmonary fibrosis. Lancet Respir Med 2013;1(3):191-192

11. Lopez-de la Mora DA, Sanchez-Roque C, Montoya-Buelna M, Sanchez-Enriquez S, Lucano-Landeros S, Macias-Barragan J, et al. Role and new insights of pirfenidone in fibrotic diseases. Int J Med Sci 2015;12(11):840-847

12. Armendáriz-Borunda J, Islas-Carbajal M, Meza-García E, Rincon AR, Lucano S, Sandoval AS, et al. A pilot study in patients with established advanced liver fibrosis using pirfenidone. Gut 2006;55:1663-1665

13. Flores-Contreras L, Sandoval-Rodríguez AS, Mena-Enriquez MG, Lucano-Landeros S, Arellano-Olivera I, Álvarez-Álvarez A, et al. Treatment with pirfenidone for 2 years decreases fibrosis, cytokine levels and enhances CB2 gene expression in patients with chronic hepatitis C. BMC Gastroenterol 2014;14:131

14. Poynard T, Vergniol J, Ngo Y, Foucher J, Munteanu M, Merrouche $\mathrm{W}$, et al. Staging chronic hepatitis $\mathrm{C}$ in seven categories using fibrosis biomarker $\left(\mathrm{FT}^{\mathrm{TM}}\right)$ and transient elastography (FibroScan ${ }^{\circledR}$ ). J Hepatol 2014;60:706-714

15. Lim JK, Flamm SL, Singh S, Falck-Ytter YT, Clinical Guidelines Committee of the American Gastroenterological Association. American Gastroenterological Association Institute Guideline on the role of elastography in the evaluation of liver fibrosis. Gastroenterology 2017;152:1536-1543

16. Cassinotto C, Lapuyade B, Mouries A, Hiriart JB, Vergniol J, Gaye D, et al. Non-invasive assessment of liver fibrosis with impulse elastography: comparison of Supersonic Shear Imaging with ARFI and FibroScan R. J Hepatol 2014;61:550-557

17. Haghgoo SM, Sharafi H, Alavian SM. Serum cytokines, adipokines and ferritin for non-invasive assessment of liver fibrosis in chronic liver disease: a systematic review. Clin Chem Lab Med 2019;57(5):577-610

18. Piotrowski D, Sączewska-Piotrowska A, Jaroszewicz J, BorońKaczmarska A. Predictive power of model for end-stage liver disease and Child-Turcotte-Pugh score for mortality in cirrhotic patients. Clin Exp Hepatol 2018;4(4):240-246

19. Faul F, Erdfelder E, Buchner A, Lang AG. Statistical power analyses using $\mathrm{G}^{*}$ Power 3.1: tests for correlation and regression analyses. Behav Res Methods 2009;41:1149-1160

20. Huang N, Ding L, Wang J, Zhang QY, Liu X, Lin HD, et al. Pharmacokinetics, safety and tolerability of pirfenidone and its major metabolite after single and multiple oral doses in healthy Chinese subjects under Fed conditions. Drug Res (Sturg) 2013;63:388-395

21. Kale V, Tharit Manoi T, Porter C, Atkinson S. Drug interaction of pirfenidone with commonly prescribed pharmaceutical drugs. FASEB J 2016;30(1):01A

22. Armendáriz-Borunda J, Rincón AR, Muñoz-Valle JF, BuenoTopete M, Oregón-Romero E, Islas-Carbajal MC, et al. Fibrogenic polymorphisms (TGF-beta, PAI-1, AT) in Mexican patients with established liver fibrosis. Potential correlation with pirfenidone treatment. J Investig Med 2008;56(7):944-953

23. Dirchwolf M, Podhorzer A, Marino M, Shulman C, Cartier M, Zunino M, et al. Immune dysfunction in cirrhosis: distinct cytokines phenotypes according to cirrhosis severity. Cytokine 2016;77:14-25

24. Meng X, Nikolic-Paterson DJ, Lan HY. TGF- $\beta$ : the master regulator of fibrosis. Nat Rev Nephrol 2016;12:325-338

25. Hartl J, Ehlken H, Sebode M, Peiseler M, Krech T, Zenouzi R, et al. Usefulness of biochemical remission and transient elastography in monitoring disease course in autoimmune hepatitis. $\mathbf{J}$ Hepatol 2018;68(4):754-763

26. Piccinino F, Sagnelli E, Pasquale G, Giusti G. Complications following percutaneous liver biopsy: a multicenter retrospective study on 68,276 biopsies. J Hepatol 1986;2:165-173

27. Li C, Li R, Zhang W. Progress in non-invasive detection of liver fibrosis. Cancer Biol Med 2018;15(2):124-136

28. Lau-Corona D, Pineda LA, Avilés HH, Gutiérrez-Reyes G, FarfanLabonne BE, Núñez-Nateras R, et al. Effective use of FibroTest to generate decision trees in hepatitis C. World J Gastroenterol 2009;15(21):2617-2622

29. Mauro Z, Crespo G, Montironi C, Londoño MC, Virginia Hernández-Gea V, Ruiz P, et al. Portal pressure and liver stiffness measurements in the prediction of fibrosis regression after sustained virological response in recurrent hepatitis C. Hepatology 2018;67(5):183-1694

30. Chalasani N, Abdelmalek MF, Loomba R, Kowdley KV, McCullough AJ, Dasarathy S, et al. Relationship between three commonly used non-invasive fibrosis biomarkers and improvement in fibrosis stage in patients with NASH. Liver Int 2019;39(5):924-932

31. Angulo P, MacCarty RL, Sylvestre PB, Jorgensen RA, Wiesner $\mathrm{RH}$, LaRusso NA, et al. Pirfenidone in the treatment of primary sclerosing cholangitis. Dig Dis Sci 2002;47:157-161

Publisher's Note Springer Nature remains neutral with regard to jurisdictional claims in published maps and institutional affiliations. 